\title{
Overshadowing in matching-to-sample: Reduction in sample-stimulus control by differential sample behaviors
}

\author{
PETER J. URCUIOLI \\ Purdue University, West Lafayette, Indiana
}

\begin{abstract}
Separate groups of pigeons were trained to high levels of accuracy on 0-delay matching-tosample with sample-response requirements that were either differential or nondifferential with respect to the sample stimuli. Differential subjects produced the comparisons by completing a differential-reinforcement-of-low-rates-of-responding 3-sec (DRL 3") requirement during one sample and a fixed-ratio (FR 10) requirement during the other. Nondifferential subjects produced the comparisons by completing the same schedule requirement (either DRL 3" or FR 10) for both samples. Following acquisition to criterion, the DRL and/or FR sample-response requirements were replaced by a nondifferential single-peck (CRF) requirement in order to assess the degree to which the samples had acquired control over choice in each group. This change disrupted performance in all subjects, but the disruption was greater for the differential birds, which generally performed at lower levels of accuracy and required more sessions of retraining to reach criterion levels of accuracy than the nondifferential birds. Follow-up experiments revealed that comparison choices by the differential birds were primarily controlled by their DRL vs. FR sample-specific behaviors. The relatively poor performance of the differential group during testing with CRF requirements suggests that the cue arising from the birds' differential sample behaviors had also overshadowed the sample stimuli for conditional control over choice. The unique, and rather unusual, aspect of this overshadowing effect is that it occurred in spite of the fact that the overshadowed cue (that provided by the samples) was necessary for producing the cue that resulted in overshadowing (the differential sample behaviors). This finding has potentially important implications for the differential outcomes effect in conditional discrimination learning and for attentional processes in compound-cue situations in general.
\end{abstract}

Most matching-to-sample (MTS) procedures require subjects to respond one or more times to each sample in order to produce the comparison stimuli. Observing responses supposedly makes it more likely that subjects will attend to the samples, thus increasing the degree to which these stimuli acquire or exert control over choice between the comparisons (Eckerman, Lanson, \& Cumming, 1968). The notion that observing primarily serves an attentional function is supported by the finding that increasing the number of required sample responses facilitates both the acquisition of 0-delay MTS (Sacks, Kamil, \& Mack, 1972) and steady-state accuracy on delayed MTS (Roberts \& Grant, 1974).

The enhancing effect of observing is particularly pronounced when subjects must respond differentially to the sample stimuli (Cohen, Looney, Brady, \& Aucella, 1976; Eckerman, 1970). For example, Cohen et al. (1976) found that pigeons that produced the comparisons by pecking

This research was supported in part by a Purdue University XL Grant. The author gratefully acknowledges Sandy Cebulis for her assistance in conducting the experiments, and Jean Cullen for typing the manuscript. Some of these data were reported at the meeting of the Midwestern Psychological Association, Chicago, May 1983. The author's mailing address is: Department of Psychological Sciences, Purdue University, West Lafayette, IN 47907. one sample on a differential-reinforcement-of-low-ratesof-responding 3-sec (DRL 3") schedule and the other sample on a fixed-ratio (FR) 16 schedule achieved $90 \%$ or better matching accuracy two to three times faster than pigeons that completed the same observing-response requirement (either DRL 3" or FR 16) for both samples. The more rapid rate of acquisition by subjects trained with differential requirements can also be interpreted as an attentional effect, especially in view of the fact that acquisition of accurate matching behavior depends upon discrimination between the successively presented samples (cf. Carter \& Eckerman, 1975). Explicit reinforcement of differential observing behavior guarantees such a discrimination (i.e., that subjects attend to the relevant characteristics of the samples) and, thus, facilitates the rate at which the samples acquire control over choice.

Urcuioli \& Honig (1980), however, have suggested another interpretation of the Cohen et al. (1976) data based upon the effects of reversing DRL 3" and FR 10 sampleresponse requirements after acquisition of MTS. They found that such a reversal caused a substantial drop in accuracy of matching by pigeons and interfered with the rate at which they relearned the matching task. On the other hand, the reversal facilitated acquisition of the opposite task, oddity-from-sample (OFS). These results, together 
with other transfer data (cf. Urcuioli \& Honig, 1980, Experiment 3 ) indicated that the differential birds had learned to choose between the comparisons primarily on the basis of their DRL vs. FR sample-specific behaviors. Reversing sample-response requirements disrupted previously learned MTS performances because the original relationship between the birds' sample-specific behaviors and correct choices had also been reversed. Conversely, OFS was acquired rapidly because this relationship was preserved by the additional reversal of correct comparisons vis-àvis the sample stimuli.

The implication drawn from these data, then, is that the facilitation in MTS acquisition reported by Cohen et al. (1976) probably occurred because different cues were controlling choices in the differential and nondifferential groups, not because of a difference in sample discriminability or attention (see also Hogan, Zentall, \& Pace, 1983). More rapid acquisition in the differential group could be explained by assuming that the cue arising from the birds' differential sample behaviors was more salient or distinctive than the cue arising from the samples themselves (cf. Lovejoy \& Russell, 1967; Miles \& Jenkins, 1973).

It is possible, however, that differential sample behaviors also increase attention to the samples in addition to providing another source of stimulus control. After all, the birds must detect (attend to) the differences between the samples in order to respond differentially to them. An attentional enhancement effect might be masked, however, by the presence of the more potent cue arising from the birds' differential sample behaviors. Alternatively, the samples might acquire less control over matching in differential than in nondifferential subjects because they are overshadowed in their control over choice by the birds' differential sample behaviors (cf. Urcuioli \& Honig, 1980). In other words, the DRL vs. FR response patterns may not only dominate control over choice but may do so at the expense of the sample stimuli that occasion them.

Experiment 1 distinguished between these alternatives by evaluating the degree to which the sample stimuli alone are able to support accurate matching in subjects trained on MTS with either differential or nondifferential sampleresponse requirements.

\section{EXPERIMENT 1}

In this experiment, separate groups of pigeons were initially trained to produce the comparisons in MTS either by responding differentially to the sample stimuli or nondifferentially to them. The degree to which the samples alone controlled the matching choices in each group was then assessed by removing the original sample-response requirements and arranging that the comparisons be produced by a single peck to each sample.

If sample-specific behaviors in the differential group increase the discriminability of or attention to the samples, then the samples alone should support higher levels of matching accuracy in differential subjects than in nondif- ferential subjects. Conversely, if differential sample behaviors overshadow the samples, then the samples alone should support lower levels of matching accuracy in differential than in nondifferential subjects.

\section{Method}

Subjects. Twelve naive White Carneaux pigeons obtained from the Palmetto Pigeon Plant (Sumter, SC) initially served as subjects. Because of equipment failures that occurred at crucial phases of the experiment, three of the original birds were replaced with additional naive subjects. Data from the replaced birds are not reported. The 12 birds participating in the complete experiment were maintained by restricted feeding at approximately $80 \%$ of their free-feeding weights throughout the study. They were housed individually in cages located in a colony room with a 14-h light-on/10-h light-off cycle. Grit and water were continuously available in the home cage.

Apparatus. The experiment was run in a single BRS/LVE pigeon chamber containing a three-key intelligence panel (Model PIP-016) and an aluminum grid floor. The pigeon's compartment measured $30.5 \times 36.8 \times 34.3 \mathrm{~cm}$. The three circular response keys on the intelligence panel were $25.4 \mathrm{~cm}$ from the grid floor and $8.3 \mathrm{~cm}$ apart centerto-center. BRS/LVE in-line projection units (Model IC-901-715) mounted behind each key provided red $(R)$ and green $(G)$ stimuli appearing as homogeneous fields. The opening for the food hopper $(5.7 \times 5.1 \mathrm{~cm})$ was positioned in the middle of the intelligence panel, equidistant from the grid floor and the center response key. A houselight (GE No. 1829 bulb), located $5.08 \mathrm{~cm}$ above the center key, provided general chamber illumination. The entire apparatus was situated in a sound-attenuating room. A blower fan mounted on the side of the chamber provided ventilation and additional masking of extraneous noises. Experimental events were controlled and monitored by a Cromemco Z-2D microcomputer.

Procedure. Birds were randomly divided into three groups $(N=4)$ and were initially trained to eat quickly and reliably from the food magazine. Birds were then shaped by successive approximations to peck the center key whenever it was lighted by a homogeneous white field. Once pecking was established to this stimulus, $R$ and $G$ hues were successively projected in random order on the center key and pecking to these stimuli was reinforced by brief access to grain. The final stage of preliminary training consisted of two 60-trial sessions during which all three keys were lighted in random order with $R$ and $G$. Food was contingent upon a single peck to the lighted key on each trial. Successive key illuminations were separated by a 10-sec intertrial interval (ITI), with no programmed consequences for dark-key pecks. The houselight remained on throughout each session.

Following magazine training and response shaping, 10 sessions of sample pretraining were conducted during which responding to the illuminated center (sample) key was reinforced on a DRL 3" and/or a FR 10 schedule. Birds in the differential group obtained food by pecking at one hue on a DRL 3" schedule and at the other hue on a FR 10 schedule. The hues and sample-response schedules were counterbalanced across subjects. Birds in the two nondifferential groups produced food by completing the same response requirement (either DRL 3 " or FR 10) for both hues. For all subjects exposed to the FR contingency, the number of responses necessary to complete the FR requirement was gradually increased from 3 (Session 1) to 5 (Sessions 2 and 3) and then to 10 (Sessions 4 through 10$)$. The DRL parameter $\left(3^{\prime \prime}\right)$ was in effect from the outset of training for all subjects exposed to this contingency. Each pretraining session contained 30 discrete-trial presentations of the $R$ and $G$ hues separated by $10-\sec$ ITIs. The order of stimulus presentation was randomized within each session with the restriction that neither hue could appear more than three times in succession. Reinforcement duration ranged from 2.0 to $3.5 \mathrm{sec}$ and was adjusted daily for each subject so as to maintain $80 \%$ body weight.

Training on 0-delay matching-to-sample (MTS) began on the session following the final day of pretraining. Each matching trial began with presentation of the $\mathbf{R}$ or $\mathbf{G}$ sample on the center key. For subjects in the differential group, completion of the DRL 3" requirement or the FR 10 requirement, depending upon the particular sample hue, immediately turned off the sample and produced $R$ and $G$ comparisons on the two adjacent side keys. For subjects in the nondifferential groups, comple- 
tion of a single requirement (either DRL 3 " or FR 10) removed the sample and produced the two comparisons. Reinforcement for all subjects was then contingent upon a single peck to the matching comparison. Pecks to the nonmatching comparison produced a time-out period (houselight turned off), the duration of which was equal to the duration of grain access on reinforced trials. The next trial then began following a 10-sec ITI.

Daily MTS sessions consisted of 100 trials. The four possible configurations of sample and comparison stimuli appeared equally often within each successive block of 20 trials, with the restriction that no configuration could appear more than three times in succession. Training for each individual bird continued until it achieved $90 \%$ or better accuracy on five of six consecutive sessions.

After reaching criterion performance, each bird was tested on 0-delay MTS with a nondifferential single-peck (CRF) requirement substituted for the DRL 3" and/or FR 10 sample-response requirements. This change permitted an assessment of the degree to which the samples alone controlled choice in the differential group by eliminating the DRL and FR sample response patterns. Furthermore, since nondifferential birds were also shifted to CRF requirements, the measure of sample-stimulus control was obtained following a comparable change for these subjects as well. Individual birds were tested daily until they again achieved $90 \%$ or better accuracy on five of six consecutive sessions, at which point testing terminated. The matching contingencies and trial orders during this phase of the experiment remained the same as they were during training.

Data analysis. Accuracy data and relative rates of matching acquisition were analyzed by evaluating sets of mutually orthogonal contrasts post hoc across the sample means using the modified Scheffe (1953) procedure recommended by Rodger (1974). The variance ratio for each contrast in the decision set was compared to new critical $F$ values, $F[E \alpha] ; v_{1}, v_{2}$ (Rodger, 1975), which control the rate of erroneous null rejections on a per-decision basis (rather than on an experimentwise basis as with Scheffe, 1953). In all analyses reported here, Type 1 error rate $(\mathrm{E} \alpha)=.05$.

\section{Results and Discussion}

Distributions of interresponse times (IRTs) for responding to each sample hue indicated that all birds quickly learned to space successive keypecks to the hue associated with the DRL contingency and to peck rapidly, without interruption, to the hue associated with the FR contingency. The percentages of IRTs greater than $1.5 \mathrm{sec}$ for the DRL hue ranged from $54 \%$ to $91 \%$ across birds on the final pretraining session, while the corresponding percentages for the FR hue ranged from $0 \%$ to $2 \%$. The schedule-appropriate response patterns indicated by these IRT data continued throughout subsequent training on MTS.

Figure 1 shows acquisition of MTS for the birds in each group. Most birds initially matched at chance levels of accuracy $(50 \%)$ but steadily improved their performance with continued training (the only notable exception being Bird C3). The rate of acquisition, however, was more rapid in the differential than in the nondifferential groups. This effect can be seen most clearly in Table 1, which shows the average numbers of sessions to the $90 \%$ accuracy criterion for each group. Statistical analyses of these data ${ }^{1}$ showed that the differential group achieved criterion in fewer sessions than the nondifferential-DRL group $[F(1,17)$ $=17.14]$. The average number of sessions to criterion in the nondifferential-FR group did not differ from the average of the corresponding numbers for the differential and nondifferential-DRL groups $[F(1,17)=1.70]$. Together, these statistical decisions imply (cf. Rodger, 1974, Equation 8 ) that the rates of acquisition were ordered from fast-

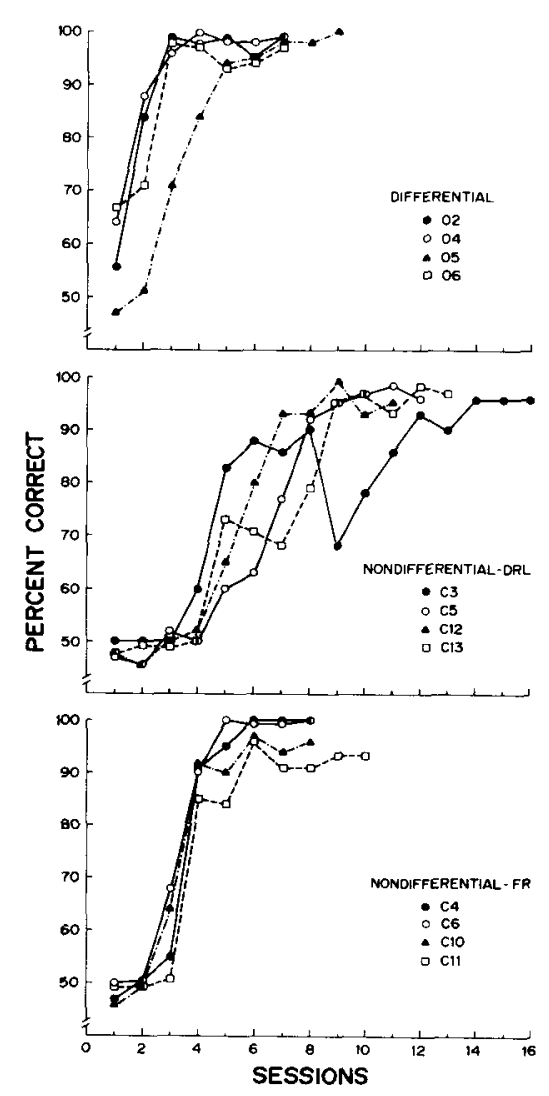

Figure 1. Mean percentage of correct choice responses during matching-to-sample acquisition for the three groups in Experiment 1.

est to slowest as follows: differential $>$ nondifferential-FR $>$ nondifferential-DRL. This pattern of results is consistent with those obtained by others using similar designs (Cohen et al., 1976; Eckerman, 1970).

Figure 2 shows that the change from DRL and/or FR requirements to the nondifferential single-peck (CRF) requirement produced a substantial drop in accuracy for subjects in all three groups. Matching performance on the first test session (top panel) averaged $68 \%, 72.5 \%, 71.8 \%$ for the differential, nondifferential-DRL, and nondifferentialFR groups, respectively. The middle and bottom panels of Figure 2 show that performance continued to deteriorate on the two subsequent test sessions for birds in the differential group, dropping to an average of $61.8 \%$ and $59.7 \%$ correct, respectively. In contrast, birds in the two nondifferential groups generally improved their performance over

Table 1

The Average Number of Sessions to the First Session of Criterion for Each Group During Training and Testing of Experiment 1

\begin{tabular}{lcc}
\multicolumn{1}{c}{ Group } & Training & Testing \\
\hline Differential & 3.5 & 10.0 \\
Nondifferential (DRL) & 9.0 & 7.5 \\
Nondifferential (FR) & 4.8 & 4.8 \\
\hline
\end{tabular}




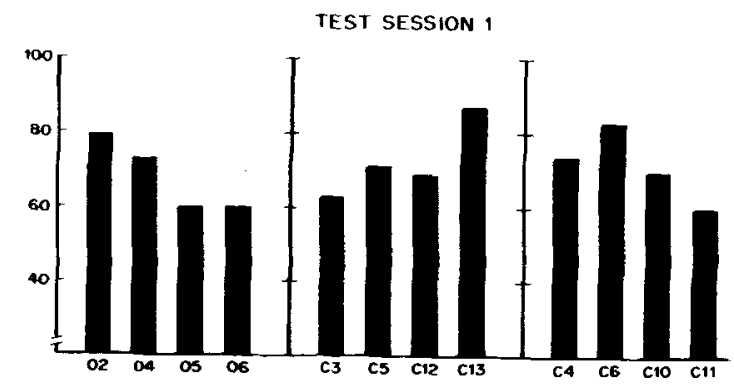

TEST SESSION 2

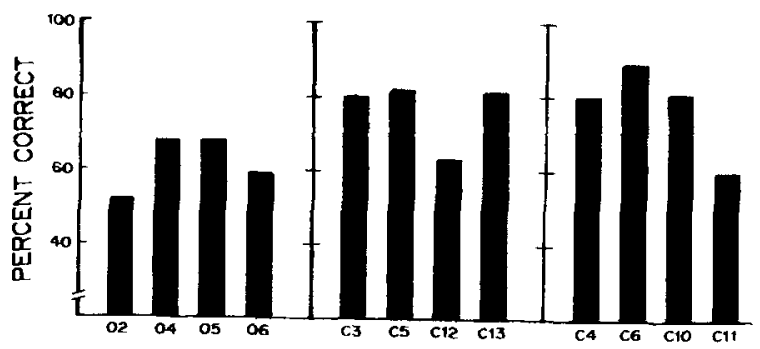

TEST SESSION 3

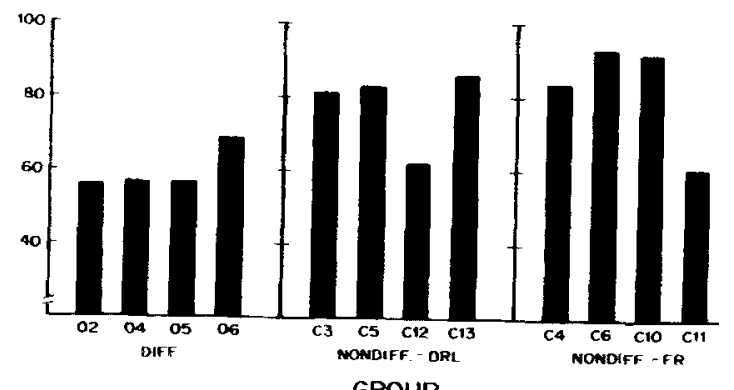

GROUP

Figure 2. Mean percentage of correct choice responses for individual subjects on the first three sessions of testing on matching-tosample with nondifferential, single-peck (CRF) requirements in Experiment 1.

these same test sessions. Accuracy in the nondifferentialDRL group increased to an average of $76.5 \%$ and $78 \%$ on Test Sessions 2 and 3, respectively. The corresponding figures for the nondifferential-FR group were $77.5 \%$ and $82.5 \%$ correct.

The average reacquisition functions for each group over the first seven sessions of testing are plotted in Figure 3 along with the average performance from the last session of training. Data from later test sessions are not shown because they involved unequal numbers of subjects per group. (Testing for each individual subject was discontinued as soon as it completed the last session of criterion.)

Statistical analyses of these data showed that the three groups did not differ in accuracy on the last session of training or on the first session of testing $[\mathrm{Fs}(1,34)<1]$, although their combined first-session test performances were clearly less accurate than those seen at the end of MTS training $[F(1,11)=118.83]$. Between-group differences did emerge, however, with continued testing. Specifically, the differential group matched at lower levels of accuracy on Test Sessions 2 and 3 than did the two nondifferential groups $[F(1,34)=7.48$ and 13.52$]$, which did not differ from one another $[\mathrm{Fs}(1,34)<1]$. On Test Sessions 4 through 6, accuracy in the differential group continued to be lower than in the nondifferential-FR group $[F s(1,34)=6.98,5.80$, and 5.80 for the three sessions, respectively], whereas accuracy in the nondifferentialDRL group did not differ from the average of the differential and nondifferential-FR groups combined [all Fs $(1,34)$ $<1]$. Together, these analyses imply that matching accuracy for the three groups on Test Sessions 4-6 was ordered from lowest to highest as follows: differential < nondifferential-DRL < nondifferential-FR. By Test Session 7 , between-group differences were no longer evident $[F(1,34)=1.64]$, probably because all subjects were at or near criterion levels of accuracy.

The test data are summarized in terms of the average numbers of sessions to criterion in the right-hand column of Table 1. Statistical analyses of the sessions-to- $90 \%$ data showed that the differential group required more sessions, on average, to reach criterion accuracy during testing than the nondifferential-FR group $[F(1,17)=15.62]$. Furthermore, the number of sessions to criterion for the nondifferential-DRL group did not differ from the average of the corresponding numbers for the differential and the nondifferential-FR groups $[F(1,17)=.00]$. These statistical decisions thus imply that reacquisition to criterion during testing was ordered from slowest to fastest as follows: differential < nondifferential-DRL < nondifferential-FR. Compared to the rates of acquisition during training, the differential group was slower to reach criterion following the shift to CRF requirements $[F(1,3)=39.0]$. The two nondifferential groups, on the other hand, achieved criterion in approximately the same number of sessions as they had during original training $[\mathrm{Fs}(1,3)<1]$.

The results of this experiment thus show that the sample stimuli did not acquire a greater degree of control over

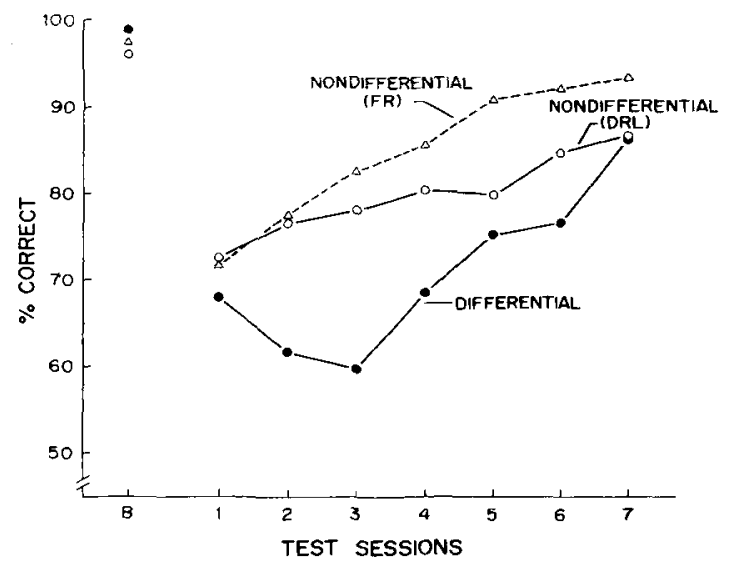

Figure 3. Mean percentage of correct choice responses during reacquisition of matching-to-sample with nondifferential, single-peck (CRF) requirements for the three groups in Experiment 1. Accuracy on the final session of training (baseline $=$ B) preceding the CRF tests is also shown for each group. 
comparison choice in birds trained with differential as opposed to nondifferential sample-response requirements. To the contrary, the samples apparently acquired less control over matching under these conditions, suggesting that they had been overshadowed in this function by the birds' differential sample behaviors. Overshadowing was implicated by the test data showing that (1) accuracy was significantly lower in differential than in nondifferential groups during individual sessions in which the birds were required to match on the basis of the samples alone, and (2) reacquisition of MTS was slower following differential than following nondifferential training.

Another way in which these differences might be explained, however, is to argue that the differential birds had simply learned during training to match on the basis of compounds consisting of the individual sample hues and the DRL vs. FR sample-specific behaviors. The change to CRF requirements during testing eliminated these compounds, which could account for their relatively poor test performances. If stimulus compounds of the type described above did indeed govern the choices of the differential birds during training, then matching accuracy should also be disrupted if the sample stimuli that occasion the DRL vs. FR response patterns are changed. Experiment 2 tested this alternative explanation.

\section{EXPERIMENT 2}

This experiment was modeled after the orthogonalsample transfer manipulation used by Urcuioli and Honig (1980, Experiment 3) to assess choice control by samplespecific behaviors in MTS. Briefly, each differential bird from Experiment 1 was trained to emit DRL and FR response patterns to two novel (line orientation) stimuli while the matching procedure was temporarily discontinued. Then, following retraining on the original MTS task, the lines were substituted for the hues as sample stimuli for MTS.

The pattern of transfer data obtained following the substitution of lines for hues indicates which particular cue controlled choices during original training. For example, if the differential birds learned to match on the basis of their DRL vs. FR sample-specific behaviors, then matching accuracy should be maintained at relatively high levels (viz, positive transfer should occur), providing that the substitution preserves the relationship between DRL vs. FR and the correct comparisons. Conversely, performances should be completely disrupted, perhaps falling to a level below chance (viz, negative transfer), if the substitution reverses this relationship. On the other hand, if the differential birds learned to match on the basis of compounds consisting of a sample hue and a samplespecific behavior, then the substitution of novel for familiar samples should disrupt performance equally under each substitution condition.

One other test was also included in Experiment 2. Since the differential birds typically took longer to complete the DRL 3" than the FR 10 requirement, the controlling cue or "contributing" element in this group may have simply been the temporal one associated with differential sample durations (cf. Stubbs, 1968). The influence of this potential temporal cue on matching was examined during a single test session in which the duration of each sample hue was equated by programming identical fixedinterval sample-response requirements.

\section{Method}

Subjects and Apparatus. The four differential birds from Experiment 1 served as subjects for this experiment. The apparatus was identical to that used previously, with the exception that two additional stimuli, three black vertical (V) stripes and three black horizontal (H) stripes on white backgrounds, were used as sample stimuli.

Procedure. Following the CRF tests of Experiment 1, each differential bird was trained over the course of five 60 -trial pretraining sessions to respond differentially to $\mathrm{V}$ and $\mathrm{H}$ lines as they appeared successively on the center key. Food reinforcement was contingent upon completion of a DRL 3" requirement in the presence of one line-orientation, and upon completion of an FR 10 requirement in the presence of the other. Lines and schedule requirements were counterbalanced across birds. The FR requirement was gradually increased across sessions from 3 (Session 1) to 5 (Sessions 2 and 3 ) and finally to 10 (Sessions 4 and 5). The 3-sec spacing requirement for the DRL schedule was in effect from the outset of pretraining. All other details of these pretraining sessions were identical to those described for the corresponding sessions in Experiment 1.

On the day following the last line-pretraining session, each bird was run for a single session on MTS with $R$ and $G$ hues and with differential sample-response requirements. This recovery session was included to insure that the birds could still match at high levels of accuracy on their original task prior to transfer. For three birds $(02,05$, and 06$)$, the recovery session was immediately followed by a series of daily test sessions during which the $V$ and $H$ lines were substituted for $R$ and $G$ as samples for MTS. The remaining bird (04) was tested with fixed-interval sampleresponse requirements in the original task (see below) prior to transfer to line samples.

The substitution of the lines for the hues for two differential birds ( 02 and 06) maintained the relationship between DRL vs. FR and the correct (hue) comparisons. The substitution reversed this relationship for the remaining two birds (04 and 05). Test sessions consisted of 100 matching trials with the order of stimulus presentation randomized in the same fashion as in Experiment 1. Each subject was run daily until it reached $90 \%$ accuracy or better on five of six consecutive sessions.

The fixed-interval (FI) test was run at different times for the three birds exposed to this particular condition (Bird 05 was not tested.) Bird 02 was tested following transfer with line samples. Bird 04 was tested following line pretraining but prior to transfer. Bird 06 received its test immediately after completing Experiment 1 (viz, prior to both line pretraining and transfer). The FI test for each bird was always preceded by a single recovery session on the original (hue sample-hue comparison) MTS task. This recovery session provided not only a necessary check on the bird's ability to continue to match accurately on its original task, but also a rough estimate of the average sample duration for the DRL and FR sample hues. Since each sample hue appeared 50 times within a session, average sample duration was based on $\mathbf{5 0}$ observations. The corresponding figures were then averaged together to provide the FI parameter for the subsequent test session. During the FI test, the birds continued to match $R$ and $G$ hues, but the comparisons were now contingent upon the first sample keypeck to occur after the programmed fixed interval had elapsed. All other matching contingencies remained the same.

\section{Results and Discussion}

The five line pretraining sessions were sufficient to establish DRL and FR behaviors to the $V$ and $H$ lines. By the end of pretraining, the line associated with the DRL requirement reliably produced spaced responding: the 
percentages of IRTs exceeding $1.5 \mathrm{sec}$ ranged from $67 \%$ to $87 \%$ across subjects. Similarly, the line associated with the FR requirement reliably produced rapid, uninterrupted responding: across birds, only $0 \%-2 \%$ of all IRTs were greater than $1.5 \mathrm{sec}$.

Figure 4 shows matching accuracy for each differential bird on the first three sessions of transfer with the novel line samples. The left-hand panel of this figure plots the transfer data for the two subjects for whom the substitution of the lines for the hues maintained the original relationship between DRL vs. FR and the correct comparisons. The right-hand panel plots the corresponding data for the two subjects for whom this relationship was reversed. Matching accuracy during the recovery (baseline) session immediately preceding the first transfer session is also shown.

Clearly, the degree/direction of transfer was affected by the manner in which the lines were substituted for the hues. Accuracy of performance was relatively unaffected (viz, positive transfer occurred) when DRL and FR were associated with the same correct choices as they had been during training (Birds 02 and 06). Both birds exposed to this transfer condition reached criterion levels of accuracy by the third transfer session. Reversing the relationship, on the other hand, produced a substantial drop in performance for the birds (04 and 05) exposed to this condition. Indeed, accuracy of matching on the very first transfer session was below the level expected by chance alone (viz, negative transfer was evident): Bird 04 matched correctly on only $39 \%$ of trials, and Bird 05 matched correctly on only $36 \%$ of all trials. Furthermore, these birds required relatively more sessions of testing to once again reach criterion: Bird 04 needed four transfer sessions, Bird 05 required nine sessions. The strong positive transfer in the "same" condition and the negative transfer in the reversed condition thus suggests that the differential birds had learned to choose between the comparisons on the basis

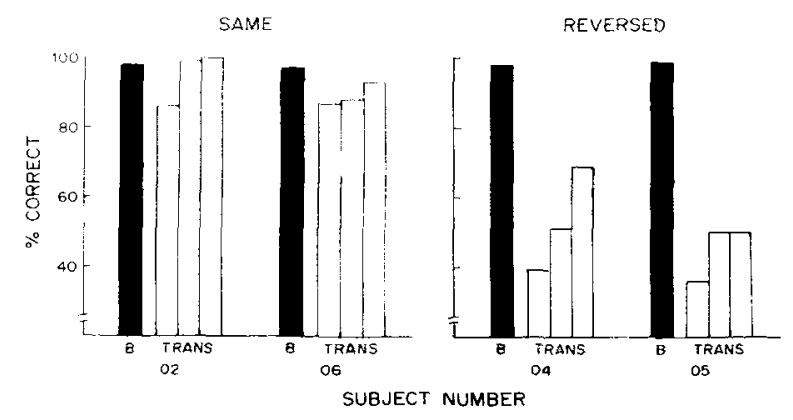

Figure 4. Mean percentage of correct choice responses for the four differential birds on the first three transfer sexions (open bars) during which vertical and horizontal lines were substituted for the red and green hues as sample stimuli for matching-to-sample. In the Same condition, the substitution of lines for hues maintained the same relationship between DRL vs. FR sample-response requirements and the correct hue comparisons that existed during training. In the reversed condition, the substitution reversed this relationship. Also shown is each bird's accuracy on the final matching-to-sample session preceding transfer (baseline $=\mathbf{B}$, filled bars).
Table 2

Choice Accuracy (in Percent) and Average Sample Durations (in Seconds) for Each Bird on the Fixed-Interval Test of Experiment 2

\begin{tabular}{cccccc} 
& \multicolumn{2}{c}{ Correct Choices } & & \multicolumn{2}{c}{ Average Sample Durations } \\
\cline { 5 - 6 } \cline { 5 - 6 } Bird No. & Baseline & Fl Test & & DRL Hue & FR Hue \\
\hline 02 & 100 & 96 & 4.8 & 4.0 \\
04 & 95 & 96 & 5.8 & 6.1 \\
06 & 97 & 96 & 7.0 & 6.5 \\
\hline
\end{tabular}

Note-Accuracy on the baseline matching task immediately preceding the test is also shown.

of their DRL vs. FR sample-specific behaviors rather than on the basis of stimulus compounds.

Table 2 shows the data from the FI test. All three birds continued to match at the same high levels of accuracy that they had exhibited when the comparisons were contingent upon completing DRL vs. FR. Furthermore, the nondifferential FI requirement was effective in removing or minimizing the difference in sample duration between the $R$ and $G$ hues, as can be seen by the average sample durations in the right-hand columns of the table. Clearly, differential sample durations did not provide a necessary temporal cue for these birds to match accurately. Instead, these data corroborate the conclusion, based on the data shown in Figure 4, that choices were controlled primarily by the bird's DRL vs. FR sample-specific behaviors. This argument is further supported by the patterns of sample responding exhibited by the differential birds during the FI test. The proportion of IRTs greater than $1.5 \mathrm{sec}$ for responding to the former DRL hue ranged from $40 \%$ to $57 \%$ across birds, showing that spaced responding appropriate to this schedule continued to occur. Conversely, the proportion of IRTs greater than $1.5 \mathrm{sec}$ for responding to the former FR hue ranged from only $1 \%$ to $4 \%$.

\section{GENERAL DISCUSSION}

The major finding of the experiments reported here is that sample-specific observing responses decreased, rather than increased, the control over choice exerted by the sample stimuli in MTS. When pigeons produced the comparisons by observing one sample on a DRL schedule and observing the other on a FR schedule, their DRL vs. FR behaviors not only acquired control over their matching performances (Experiment 2) but did so at the expense of the sample stimuli that occasioned them (Experiment 1). This latter finding indicates that the birds' differential sample behaviors overshadowed the sample stimuli for the control over choice. This effect was demonstrated by removing the cue associated with the differential behaviors (via the change to CRF sample-response requirements), so that the samples provided the only available cue for choice. Under these test conditions, the differential birds matched at lower levels of accuracy and required more sessions of retraining to reach criterion levels of accuracy than did birds that had been trained with nondifferential requirements. Thus, although samples provided a valid 
choice cue for both differential and nondifferential groups during training, these stimuli acquired substantially less control over matching when an equally valid (i.e., redundant) cue arising from the birds' differential sample behaviors was also present.

The current study differs in a number of respects from traditional studies of overshadowing (e.g., Farthing, 1972; Kamin, 1969; Kehoe, 1982). The first difference is a procedural one: the change in sample-response requirements for all groups during testing is somewhat of a departure from the usual design (cf. Mackintosh, 1974). Typically, single-stimulus groups (corresponding to the nondifferential groups here) experience little or no change in their conditioned stimuli in the shift from training to testing, whereas compound-stimulus groups encounter a major change when one element of their compound is removed during testing. As a result, poorer test performances by subjects initially trained with a compound CS can also be explained by generalization decrement, without any additional appeal to overshadowing. A similar argument applied here must assume that the change in CRF requirements altered some important aspect of the sample stimuli to a greater extent in the differential than in the nondifferential groups. For example, if sample durations decreased relatively more in the differential group, then generalization decrement from this source might explain their poorer matching performances during testing (cf. Roberts \& Grant, 1974). However, average sample durations (timed from the first to the last sample keypeck) were reduced at least as much, and in some cases more, in the nondifferential-DRL group than in the differential group, yet the former was more accurate and required fewer sessions of retraining to reach criterion accuracy during testing than did the latter. Thus, the test data cannot be easily explained by generalization decrement due to a differential change in sample duration.

Of course, other sources of generalization decrement are not so easily dismissed. For example, perhaps the shift from two different sample-response patterns (DRL and FR) to one (CRF) in the differential group produced more disruption than did the shift from one response pattern (either DRL or FR) to another (CRF) in the nondiffferential groups. Designs more complicated than the one used here (see, e.g., Wagner, Logan, Haberlandt, \& Price, 1968) are necessary to rule out factors such as these. For the present purposes, however, it is more important to note that at least some sources of generalization decrement were controlled in the current experiment.

A second difference is that one of the cues in the sample compound was provided by the animal's own differential behavior. Typically, both Pavlovian and instrumental studies of overshadowing employ only exteroceptive cues which can be directly controlled by the experimenter (e.g., Kamin, 1969; Lovejoy \& Russell, 1967; Mackintosh, 1976; Miles \& Jenkins, 1973). Recent evidence in the literature, however, suggests that stimuli associated with an animal's responses can overshadow or be overshadowed by extero- ceptive cues (Garrud, Goodall, \& Mackintosh, 1981; Hall, 1982; St. Claire-Smith, 1979). For example, Pearce and Hall (1978, Experiment 5) and Hall (1982) have shown that response rates on variable-interval (VI) schedules of food reinforcement are lower when a response-contingent stimulus (e.g., a flash of light) immediately precedes delivery of food than when it is uncorrelated with reinforcer delivery. They interpret this effect as evidence that VI responding can be overshadowed (and thus reduced) by an exteroceptive stimulus that is a better predictor of food (cf. Wagner et al., 1968). In the present experiment, sample-response patterns and sample stimuli were equally valid predictors of the reinforcer-procuring choice response. Under these conditions, it was the exteroceptive cue which was overshadowed by the response-produced cue, apparently because the latter was more salient than the former (cf. Mackintosh, 1976). Whether or not this effect might be overridden or reversed if the less salient samples were better predictors of correct choices is a question for future research.

A third difference is that the redundant elements comprising the sample compound were not independent of one another. Usually, either element can be manipulated or removed without altering the remaining one. In the matching procedure, however, the $R$ and $G$ samples were necessary for the DRL and FR response patterns to occur: eliminating the former would certainly have eliminated the latter. In spite of this dependency, the bird's differential sample behaviors still overshadowed the sample stimuli for the control over choice. This seems somewhat paradoxical, considering that the birds had to attend to the samples in order to execute the appropriate sample-specific behaviors. Why, then, were the samples alone unable to support accurate matching performances?

One explanation is that the effect seen here does not really reflect distributed attention or stimulus competition between two simultaneous cues (cf. James \& Wagner, 1980; Rescorla \& Wagner, 1972) but, rather, is a form of serial learning or chaining (Riley, Cook, \& Lamb, 1981). Since the DRL and FR behaviors were initiated some time after the samples had appeared, they may have dominated control over matching simply because they occurred closer in time to the choice point. This form of "sequential" overshadowing (Mackintosh \& Reese, 1979) might also explain why pigeons who develop sample-specific mediating behaviors during the retention interval of delayed MTS are unable to maintain high levels of matching accuracy when these behaviors are disrupted (Blough, 1959). In order for this analysis to be applicable to the present situation, however, it must virtually ignore the fact that the samples and the sample-response patterns overlapped and terminated simultaneously, even though the former slightly preceded the latter. The explanation could still remain viable, of course, if it could be shown that the samples were responsible only for initiating the sample-response patterns. However, unpublished data from my laboratory indicates that the samples must be present after the first peck of the 
sample-response pattern has been executed, or else the latter deteriorates. Thus, the samples and the DRL vs. FR patterns do not give rise to cues occurring in a nonoverlapping, serial fashion.

An alternative explanation is that the MTS task for the differential birds really consisted of two independent discrimination problems, the first involving execution of the appropriate sample-specific behavior and the second involving the matching (choice) discrimination. The $R$ vs. $G$ sample hue provided the only cue for the first discrimination, but two cues (the samples and the birds' DRL vs. FR sample behaviors) were available to solve the second. For the latter discrimination, the birds apparently attended selectively to the more salient cue arising from their differential sample behaviors (cf. Mackintosh, 1976; Urcuioli \& Honig, 1980). If this explanation is correct, then the present results also suggest that overshadowing can occur even when subjects are known to have previously attended to the overshadowed cue within the trial itself. This would seem to be at odds with certain theoretical views (e.g., Mackintosh, 1975) that claim that overshadowing occurs because subjects learn to ignore a less salient, or less valid, cue (in this instance, the samples).

Although the exact mechanism(s) underlying the present results remain unclear, the sort of interaction observed here between the samples and the birds' differential sample behaviors may also occur between samples and reward expectancies in the differential outcomes paradigm (e.g., Brodigan \& Peterson, 1976; Delong \& Wasserman, 1981; Peterson, Wheeler, \& Trapold, 1980). This paradigm, as used with MTS, involves presenting a unique reward for correct choices on each type of matching trial. For example, food might follow the (correct) selection of the red comparison on red-sample trials, whereas water would follow selection of the green comparison on green-sample trials. The effect of differential outcomes is to facilitate MTS acquisition and improve memory performance on delayed MTS relative to nondifferential outcomes.

One interpretation of these data is that subjects form differential reward expectancies on the basis of the unique sample-outcome relationships and that these expectancies control, at least in part, subjects' choices between the comparisons (Honig, Matheson, \& Dodd, 1984; Peterson \& Trapold, 1980; Peterson, Wheeler, \& Armstrong, 1978). Indeed, pigeons show positive transfer of performance when novel samples paired with the same reward outcomes are substituted for the samples used in original training (Edwards, Jagielo, Zentall, \& Hogan, 1982; Honig et al., 1984; Peterson \& Trapold, 1980). Conversely, transfer is either absent or negative when novel samples are paired with the opposite outcomes (Edwards et al., 1982). If reward expectancies operate by providing a redundant cue for choice, then perhaps they also overshadow the samples. The finding that matching performances deteriorate dramatically when birds initially trained with differential outcomes are shifted to nondifferential outcomes (Honig et al., 1984; Peterson et al., 1978) is consistent with this analysis.
There are a number of similarities between the differential outcomes paradigm and the differential sampleresponse paradigm which encourage the view that similar stimulus interactions may be occurring during the sample presentation period. For example, reward expectancies (like DRL vs. FR sample behaviors) depend upon discriminating between the samples, or else the bird would be unable to determine which outcome was contingent upon a correct choice. Reward expectancies must also begin some time after the animal views the sample, and probably persist at least as long as the sample is present. Finally, in some (but not all) cases, differential outcomes generate differential sample behaviors-for instance, different sample-keypeck topographies associated with food and water reinforcers (Brodigan \& Peterson, 1976; Jenkins $\&$ Moore, 1973). If these similarities are indicative of similar stimulus interactions between redundant conditional cues, then the differential sample-response paradigm may provide a particularly helpful method for studying the nature of these interactions, since the source of the redundant cue is overt and clearly manipulable when birds are explicitly trained to observe the samples differently.

\section{REFERENCES}

Blougr, D. (1959). Delayed matching in the pigeon. Journal of the Experimental Analysis of Behavior, 2, 151-160.

Brodigan, D. L., \& Peterson, G. B. (1976). Two-choice conditional discrimination performance of pigeons as a function of reward expectancies, prechoice delay, and domesticity. Animal Learning \& Behavior, 4, 121-124.

Carter, D. E., \& Eckerman, D. A. (1975). Symbolic matching by pigeons: Rate of learning complex discriminations predicted from simple discriminations. Science, 187, 662-664.

Cohen, L. R., Looney, T. A., Brady, J. H., \& Aucella, A. F. (1976). Differential sample response schedules in the acquisition of conditional discriminations by pigeons. Journal of the Experimental Analysis of Behavior, 26, 301-314.

Delong, R. E., \& Wasserman, E. A. (1981). Effects of differential reinforcement expectancies on successive matching-tosample performance in pigeons. Journal of Experimental Psychology: Animal Behavior Processes, 7, 394-412.

Eckerman, D. A. (1970). Generalization and response mediation of a conditional discrimination. Journal of the Experimental Analysis of Behavior, 13, 301-316.

Eckerman, D. A., Lanson, R. N., \& Cumming, W. W. (1968). Acquisition of matching without a required observing response. Journal of the Experimental Analysis of Behavior, 11, 435-441.

Edwards, C. A., Jaghelo, J. A., Zentall, T. R., \& Hogan, D. E. (1982). Acquired equivalence and distinctiveness in matching to sample by pigeons: Mediation by reinforcer-specific expectancies. Journal of Experimental Psychology: Animal Behavior Processes, 8, 244-259.

Fartilne, G. W. (1972). Overshadowing in the discrimination of successive compound stimuli. Psychonomic Science, 28, 29-32.

Garkud, P., Goodall, G., \& Mackintosh, N. J. (1981). Overshadowing of a stimulus-reinforcer association by an instrumental response. Quarterly Journal of Experimental Psychology, $33,123-135$.

HaLl, G. (1982). Effects of a brief stimulus accompanying reinforcement on instrumental responding in pigeons. Learning and Motivation, 13, 26-43.

Hogan, D. E., Zentall, T. R., \& Pace, G. (1983). Control of pigeons' matching-to-sample performance by differential 
sample response requirements. American Journal of Psychology, 96, 37-49.

Honig, W. K., Matheson, W. R., \& Dodd, P. W. D. (1984). Outcome expectancies as mediators for discriminative responding. Canadian Journal of Psychology, 38, 196-217.

JAMES, J. H., \& WAGNER, A. R. (1980). One-trial overshadowing: Evidence of distributive processing. Journal of Experimental Psychology: Animal Behavior Processes, 6, 188-205.

Jenkins, H. M., \& MoORE, B. R. (1973). The form of the autoshaped response with food or water reinforcer. Journal of the Experimental Analysis of Behavior, 20, 163-181.

KAMIN, L. J. (1969). Selective association and conditioning. In N. J. Mackintosh \& W. K. Honig (Eds.), Fundamental issues in associative learning (pp. 42-64). Halifax: Dalhousie University Press.

KeноE, E. J. (1982). Overshadowing and summation in compound stimulus conditioning of rabbit's nictitating membrane response. Journal of Experimental Psychology: Animal Behavior Processes, 8, 313-328.

Lovejoy, E., \& Russell, D. G. (1967). Suppression of learning about a hard cue by the presence of an easy cue. Psychonomic Science, 8, 365-366.

Mackintosh, N. J. (1974). The psychology of animal learning. New York: Academic Press.

Mackintosh, N. J. (1975). A theory of attention: Variations in the associability of stimuli with reinforcement. Psychological Review, 82, 276-298.

Mackintosh, N. J. (1976). Overshadowing and stimulus intensity. Animal Learning \& Behavior, 4, 186-192.

Mackintosh, N. J., \& ReEse, B. (1979). One-trial overshadowing. Quarterly Journal of Experimental Psychology, 31, 519-526.

Miles, C. G., \& Jenkins, H. M. (1973). Overshadowing in operant conditioning as a function of discriminability. Learning and Motivation, 4, 11-27.

Pearce, J. M., \& Hall, G. (1978). Overshadowing the instrumental conditioning of a lever-press response by a more valid predictor of reinforcement. Journal of Experimental Psychology: Animal Behavior Processes, 4, 356-367.

Peterson, G. B., \& Trapold, M. A. (1980). Effects of altering outcome expectancies on pigeons' delayed conditional discrimination performance. Learning and Motivation, 11, 267-288.

Peterson, G. B., Wheeler, R. L., \& Armstrong, G. D. (1978). Expectancies as mediators in differential-reward conditional discrimination performance of pigeons. Animal Learning \& Behavior, 6, 279-285.

Peterson, G. B., Wheeleh, R. L., \& Trapold, M. A. (1980). Enhancement of pigeons' conditional discrimination performance by expectancies of reinforcement and nonreinforcement. Animal Learning \& Behavior, 8, 22-30.

Rescorla, R. A., \& Wagner, A. R. (1972). A theory of Pavlovian conditioning: Variations in the effectiveness of reinforcement and nonreinforcement. In A. H. Black \& W. F. Prokasy (Ed.), Classical conditioning II: Theory and research (pp. 64-99). New York: Appleton-Century-Crofts.

Riley, D. A., Cook, R. G., \& Lamb, M. R. (1981). A classification and analysis of short-term retention codes in pigeons.
In G. S. Bower (Ed.), The psychology of learning and motivation (Vol. 15, pp. 51-79). New York: Academic Press.

Roberts, W. A., \& Grant, D. S. (1974). Short-term memory in the pigeon with presentation time precisely controlled. Learning and Motivation, 5, 393-408.

RODGER, R. S. (1974). Multiple contrasts, factors, error rate and power. British Journal of Mathematical and Statistical Psychology, 27, 179-198.

Rodger, R.S. (1975). The number of non-zero, post-hoc contrasts from ANOVA and error rate. I. British Journal of Mathematical and Statistical Psychology, 28, 71-78.

Sacks, R. A., Kamil, A. C., \& Mack, R. (1972). The effects of fixed-ratio sample requirements on matching to sample in the pigeon. Psychonomic Science, 36, 291-293.

Satterthwaite, F. E. (1946). An approximate distribution of estimates of variance components. Biometrics Bulletin, 2 , 110-114.

Scheffé, H. (1953). A method for judging all contrasts in the analysis of variance. Biometrika, $40,87-104$.

St. Claire-Smith, R. (1979). The overshadowing of instrumental conditioning by a stimulus that predicts reinforcement better than the response. Animal Learning \& Behavior, 7, 224-228.

StuBbs, D. A. (1968). The discrimination of stimulus duration by pigeons. Journal of the Experimental Analysis of Behavior, 11, 223-238.

Urcuioli, P. J., \& Honig, W. K. (1980). Control of choice in conditional discriminations by sample-specific behaviors. Journal of Experimental Psychology: Animal Behavior Processes, 6, 251-277.

Wagner, A. R., Logan, F. A., Haberlandt, K., \& Paice, T. (1968). Stimulus selection in animal discrimination learning. Journal of Experimental Psychology, 76, 171-180.

\section{NOTE}

1. The error mean square used for this analysis (and for the subsequent analysis of the test data) was the average of the two individual error mean squares obtained from separate one-way ANOVAs on the sessions-to-90\% data for training and testing. This averaged or common error term provides a better estimate of the true population variance than is provided by the individual error terms from the separate one-way ANOVAs and was used for this reason. However, because correlated observations are involved in this method of analysis, the degrees of freedom for the common error term is not simply the average of those for the individual error terms. Rather, the degrees of freedom must be modified according to the formula provided by Satterthwaite (1946). The results of this calculation appear as the reported error degrees of freedom.

The procedure of using a common error mean square for analyses of between-group differences over successive treatment conditions or days was also followed in the statistical analysis of the data shown in Figure 3 .

(Manuscript received October 28, 1983; revision accepted for publication April 22, 1984.) 